\title{
Phases of two-dimensional spinless lattice fermions with first-quantized deep neural-network quantum states
}

\author{
James Stokes $\odot,,^{1,2, *, \dagger}$ Javier Robledo Moreno $\odot,{ }^{1,3, *, *}$ Eftychios A. Pnevmatikakis $\oplus^{2, \S}$ and Giuseppe Carleo ${ }^{4, \|}$ \\ ${ }^{1}$ Center for Computational Quantum Physics, Flatiron Institute, New York, New York 10010, USA \\ ${ }^{2}$ Center for Computational Mathematics, Flatiron Institute, New York, New York 10010, USA \\ ${ }^{3}$ Center for Quantum Phenomena, Department of Physics, New York University, 726 Broadway, New York, New York 10003, USA \\ ${ }^{4}$ Institute of Physics, École Polytechnique Fédérale de Lausanne (EPFL), CH-1015 Lausanne, Switzerland
}

(Received 17 August 2020; accepted 27 October 2020; published 20 November 2020)

\begin{abstract}
First-quantized deep neural network techniques are developed for analyzing strongly coupled fermionic systems on the lattice. Using a Slater-Jastrow-inspired ansatz which exploits deep residual networks with convolutional residual blocks, we approximately determine the ground state of spinless fermions on a square lattice with nearest-neighbor interactions. The flexibility of the neural-network ansatz results in a high level of accuracy when compared with exact diagonalization results on small systems, both for energy and correlation functions. On large systems, we obtain accurate estimates of the boundaries between metallic and charge-ordered phases as a function of the interaction strength and the particle density.
\end{abstract}

DOI: 10.1103/PhysRevB.102.205122

\section{INTRODUCTION}

The difficulty in treating interacting quantum systems stems directly from the fact that the state space of a manybody quantum system grows exponentially with the number of its constituents. The quantum many-body problem is a severe bottleneck in the understanding of complex quantum phenomena in many domains of science where quantum effects are relevant. When many-body effects are dominant, variational methods have proven a successful strategy to approximately represent, in a compact and computationally manageable form, many-body quantum states.

The prevailing paradigm for simulating lattice quantum systems in one spatial dimension is the density-matrix renormalization group (DMRG) [1,2], which involves an iterative procedure to approximate low-entanglement quantum states using representations known as matrix product states $[3,4]$. The success of DMRG to produce high overlap with the ground space stems from the ability of matrix product states to approximate gapped one-dimensional quantum systems [5] and the existence of a very efficient numerical scheme for their variational optimization. In two or more spatial dimensions, however, the situation is qualitatively very different and research into both computationally efficient and compact

\footnotetext{
*J.S. and J.R.M. contributed equally to this work.

†jstokes@flatironinstitute.org

¥jrm874@nyu.edu

§epnevmatikakis@flatironinstitute.org

" giuseppe.carleo@epfl.ch
}

Published by the American Physical Society under the terms of the Creative Commons Attribution 4.0 International license. Further distribution of this work must maintain attribution to the author(s) and the published article's title, journal citation, and DOI. variational representations of quantum ground states is very active.

A different context which directly confronts the curse of dimensionality is machine learning, which has recently found several applications in physics problems where highdimensional functions are to be approximated [6]. The ability of generative models based on neural networks to overcome the curse of dimensionality in a variety of learning problems has motivated the development of neural network quantum states (NQSs) and an associated real- or imaginary-time evolution algorithm [7] that extends the scope of the variational Monte Carlo [8] to a number of challenging two-dimensional lattice systems.

Neural-network-based variational simulation has predominantly focused on systems corresponding to strongly localized electrons in which all spatial degrees of freedom have been frozen out, leaving an effective lattice Hamiltonian governing the spin degrees of freedom [7,9-12].

Neural networks have also recently been proposed for simulating fermionic systems in the second-quantized formalism [13]. This approach involves mapping the fermionic modes to an interacting quantum spin model, for example, through a Jordan-Wigner transformation. The reduction of the fermionic Hamiltonian to a spin model makes it possible to capitalize on the successes of NQSs for spin systems, but suffers from the disadvantage that the resulting spin Hamiltonian is nonlocally interacting. First quantization is an attractive alternative formalism, which preserves the locality of the physical interactions. In first quantization, the solution of the quantum fermionic many-body problem can be posed as a function approximation problem, in which the target function to be approximated is a totally antisymmetric solution of the time-independent Schrödinger equation. First quantization has been explored for spinful Hubbard Hamiltonians, predominantly focusing on restricted Boltzmann machines [14], Pfaffian states [15], and backflow transformations [16]. This 
approach has also been applied to $a b$ initio calculations of interacting electrons in the continuum $[17,18]$. In this paper, we focus on the problem of approximating the ground state for a model of two-dimensional spinless fermions with nearestneighbor interactions, modeling the wave function using a Slater-Jastrow-inspired factorization [19], with an additional neural network trained to capture sign deviations [20] compared with the Slater determinant.

The paper is organized as follows: we begin by introducing the Hamiltonian, the qualitative features of the phase structure, and the observables that have been considered for identifying phase boundaries, as well as our proposed order parameter. We then discuss the detailed optimization problem and the relationship between variational Monte Carlo and other variational methods such as Hartree-Fock. Finally, we present results for the phase structure and ground-state correlation functions.

\section{THEORY}

\section{A. States and Hamiltonian}

Consider a system of spinless fermions hopping on the edges of a simple undirected graph $G=(\mathcal{V}, \mathcal{E})$ with vertices $\mathcal{V}$ and edges $\mathcal{E}$. By Fermi statistics the number of fermions is constrained by $N \in\{0,1, \ldots,|\mathcal{V}|\}$. For a $d$-dimensional hypercubic lattice with periodically identified boundaries, we have $|\mathcal{V}|=L^{d}$, where $L$ is the side length of the lattice. The particle density is denoted by $\bar{n}=N /|\mathcal{V}|$. Let $\mathcal{V}^{N}$ be the set of $N$ tuples $x=\left(i_{1}, \ldots, i_{N}\right) \in \mathcal{V}^{N}$ and let $\mathcal{A}_{N}$ denote the complex vector space of totally antisymmetric functions mapping $\mathcal{V}^{N} \rightarrow \mathbb{C}$, which is of dimension $\operatorname{dim}_{\mathbb{C}} \mathcal{A}_{N}=C_{N}^{|\mathcal{V}|}$.

To describe the action of the Hamiltonian on the Hilbert space of states, we employ the following Fock-space construction, which is reviewed in the Appendixes. Fix an ordering $\leqslant$ on the vertices $i \in \mathcal{V}$. Then an orthonormal basis of $N$ particle states is given by $|x\rangle:=\hat{c}_{i_{1}}^{\dagger} \cdots \hat{c}_{i_{N}}^{\dagger}|0\rangle$ with the $N$ tuples $x=\left(i_{1}, \ldots, i_{N}\right)$ restricted by the condition $i_{1}<\cdots<i_{N}$ and where $|0\rangle$ denotes the Fock vacuum, which is annihilated by all $\hat{c}_{i}$. The associated Hilbert space $\mathcal{H}_{N}=\operatorname{span}_{\mathbb{C}}\left\{|x\rangle: i_{1}<\right.$ $\left.\cdots<i_{N}\right\}$ is isomorphic to $\mathcal{A}_{N}$. A general state vector $|\Psi\rangle \in$ $\mathcal{H}_{N}$ can be expanded over the basis as

$$
|\Psi\rangle=\sum_{i_{1}<\cdots<i_{N}}\langle x \mid \Psi\rangle|x\rangle=: \sum_{x \in \mathcal{V}^{N}} f(x)|x\rangle,
$$

where in the second equality we have used the Fermi algebra to expand $|\Psi\rangle$ over a spanning set, with coefficients given by the output of the antisymmetric function $f \in \mathcal{A}_{N}$ defined by $f(x):=(1 / N !)\langle x \mid \Psi\rangle$. Conversely, any antisymmetric function $f \in \mathcal{A}_{N}$ gives rise to a valid state vector. This paper focuses on variational families of states in which the antisymmetric function $f \in \mathcal{A}_{N}$ is modeled by using a parametrized neural network.

The hopping dynamics on the graph $G=(\mathcal{V}, \mathcal{E})$ is parametrized in terms of a pair of coupling parameters $t, V \geqslant$ 0 , which represent the kinetic energy and repulsive interaction strength, respectively,

$$
\hat{H}=\sum_{\{i, j\} \in \mathcal{E}}-t\left(\hat{c}_{i}^{\dagger} \hat{c}_{j}+\hat{c}_{j}^{\dagger} \hat{c}_{i}\right)+V \hat{n}_{i} \hat{n}_{j},
$$

where $\hat{n}_{i}=\hat{c}_{i}^{\dagger} \hat{c}_{i}$. The above Hamiltonian commutes with the fermion number operator $\hat{N}=\sum_{i \in \mathcal{V}} \hat{n}_{i}$, making it possible to restrict to the fixed particle number subspace $\mathcal{H}_{N}$.

This model Hamiltonian of spinless fermions in two dimensions is approximately realized in several systems of physical interest, including adsorbed submonolayers of spinpolarized ${ }^{3} \mathrm{He}_{\downarrow}$ and $D_{\downarrow}$ [21,22], several organic materials (at one-quarter filling) [23], or ultracold atomic gases of spin-polarized ${ }^{6} \mathrm{Li}[24,25]$. The model is believed to exhibit a nontrivial phase transition between metallic and chargeordered phases. Despite its apparent simplicity, however, the phase diagram cannot be determined with high precision using existing numerical approaches and several questions remain open. These include the nature of the charge-ordered phase [26,27] and the precise position of the phase boundary [21,22,26-29]. Several state-of-the-art variational wave functions have been applied to solve this model, including the so-called string-bond states [28] and tensor-product projected states [29] (at half filling), as well as fermionic projected entangled-pair states (IPEPS) [30] at arbitrary filling in the grand canonical ensemble. In this work we use neural network states to systematically investigate the phase diagram in the fixed particle subspace by considering a large collection of model parameters.

\section{B. Phase structure}

Throughout this section, we focus exclusively on the $L \times L$ square with periodic boundary conditions. Moreover, we only consider even values of the side length $L$ such that $L^{2}$ is even so that the lattice supports half occupation. A rough picture of the $\bar{n}-V / t$ phase diagram can be determined by considering the limits of strong coupling $(V / t \rightarrow \infty)$ and weak coupling $(V / t \rightarrow 0)$, in which the model becomes exactly soluble for any value of $\bar{n}$. In the strong-coupling limit $V / t \rightarrow \infty$, the system behaves like a hard-core classical lattice gas, in which translation invariance is broken as a result of charge ordering. In the particular case of half occupation $(\bar{n}=0.5)$ the charge ordering is a staggered, checkerboard pattern and the corresponding phase is insulating. The noninteracting limit $V / t \rightarrow 0$ exhibits uniform density distribution and powerlaw density-density correlation functions, characteristic of a metallic phase. The above phase structure is qualitatively similar in one spatial dimension, where the phase diagram can be exactly computed by using the Bethe ansatz and bosonization [31].

Although the phase diagram has been investigated using a variety of techniques [21,22,26-30,32], there still exist open questions concerning the precise location of the phase boundary (particularly at half occupation $[21,22,28,29]$ ), as well as the nature of the charge-ordered phase in the vicinity of the critical point [27].

An unrestricted Hartree-Fock (HF) analysis [26] found that, for sufficiently small values of the interaction, the system is in a gapless metallic state with uniform charge distribution. A critical value of $V / t$ was found, above which a first-order phase transition leads to a thermodynamically unstable phase separation state where the system is comprised of both metallic and staggered charge-density wave components. More recently [27], an analysis using mean-field antinodal fermions 
refuted the hypothesis of a phase separation state, which was claimed to be an artifact of lack of accuracy of the unrestricted Hartree-Fock approximation, in a region of the phase diagram where the system is highly degenerate. They argue that the true nature of this state is a gapped, symmetry-broken charge-density wave (CDW) state with commensurate (staggered) charge order at half occupation and incommensurate charge order otherwise. Both of these analyses are formulated in the grand canonical ensemble, where the Hilbert space is chosen by the unrestricted Fock space, and the Hamiltonian is parametrized by a chemical potential $\mu \in \mathbb{R}$ as $\hat{H}(\mu)=$ $\hat{H}-\mu \hat{N}$. The phase diagram was constructed by identifying cusps in the ground-state energy of $\hat{H}(\mu)$ as a function of $\mu$. In contrast, since we restrict to a subspace $\mathcal{H}_{N}$ defined by fixed particle number, the chemical-potential term only contributes an irrelevant constant to the energy, so we require a different strategy to find the transition points.

Different observables have been considered for detecting the phase transition at fixed particle number. In particular, at half-occupation $(\bar{n}=0.5)$, the phase transition from a metallic phase to a checkerboard charge-ordered insulating phase is detected by the so-called charge structure factor $S(\pi, \pi)$, defined as the $\boldsymbol{k}=(\pi, \pi)$ component of the Fourier transform of the two-point correlation function averaged over lattice locations [21,22,28,29].

This observable abruptly increases upon crossing to the charge-ordering phase due to the staggered charge ordering $[21,22]$. The charge structure factor $S(\pi, \pi)$ is not suitable for identifying the transition away from half filling because it assumes that the charge ordering is commensurate with the underlying lattice.

To define an order parameter suitable for general filling fraction, we first identify the vertex set with the twodimensional periodic torus $\mathcal{V} \cong \mathbb{Z}_{L}^{2}$ (where $\mathbb{Z}_{L}=\{0, \ldots, L-$ 1\}) and define a density signal $\rho: \mathbb{Z}_{L}^{2} \rightarrow[0,1]$ on the torus by $\rho(\boldsymbol{r})=\left\langle\hat{n}_{\boldsymbol{r}}\right\rangle$ where $\boldsymbol{r} \in \mathbb{Z}_{L}^{2}$. We seek an order parameter $o$ that measures the departure of this density signal from homogeneity so we define

$$
o[\rho]:=\frac{L^{2}}{N\left(L^{2}-N\right)}\left[\|\rho\|_{2}^{2}-\frac{1}{L^{2}}\|\rho\|_{1}^{2}\right] \geqslant 0 .
$$

Positivity of the order parameter follows directly from the relation between the $l_{1}$ and $l_{2}$ norms. The bound is saturated when $\rho$ is a constant function (uniform density) and the overall normalization is chosen such that the order parameter is unity for a classical $N$-particle state. Moreover, by Parseval's identity, this order parameter is related to the energy in the nonzero Fourier modes

$$
o[\rho]=\frac{1}{N\left(L^{2}-N\right)} \sum_{\boldsymbol{k} \neq \mathbf{0}}|\widetilde{\rho}(\boldsymbol{k})|^{2},
$$

where the $\boldsymbol{k}$ sum is over all nonzero modes in the discrete Brillouin torus $\frac{2 \pi}{L} \mathbb{Z}_{L}^{2}$. This quantity is evidently dependent on multiple Fourier modes, as required to capture incommensurate order. Therefore, in the thermodynamic limit, this observable vanishes in the metallic phase and becomes nonzero and finite upon the formation of a charge-ordered state.
Another observable which has received attention in this context is the so-called density-density correlation function, which is defined for ${ }^{1} r \in \mathbb{Z}_{L+1}=\{0, \ldots, L\}$ as follows:

$$
C(r)=\frac{1}{L^{2} N_{r}} \sum_{i \in \mathcal{V}} \sum_{j \in S_{r}(i)}\left\langle\left(\hat{n}_{i}-\bar{n}\right)\left(\hat{n}_{j}-\bar{n}\right)\right\rangle,
$$

where $S_{r}(i)=\{j \in \mathcal{V}: d(i, j)=r\}$ is the set of vertices with graph distance $r$ from $i \in \mathcal{V}$ and $N_{r}=\left|S_{r}(i)\right|$ is the number of such vertices, which is constant for the square lattice under consideration.

Due to the expected short-distance divergences of the twopoint correlation function in the continuum limit, we define the renormalized Fourier space correlator by subtracting the coincidence limit of the position space correlator

$$
\widetilde{C}_{\mathrm{ren}}(k):=\sum_{r=0}^{L} e^{-i k r} C_{\mathrm{ren}}(r)=\sum_{r=1}^{L} e^{-i k r} C(r),
$$

where $k \in \frac{2 \pi}{L+1}\{0, \ldots, L\}$.

\section{METHODS}

In this section we describe the variational Monte Carlo and Hartree-Fock optimization problems and our proposed variational ansatz.

\section{A. Optimization problem}

For each integer particle number $N$ in the range $1 \leqslant N \leqslant$ $|\mathcal{V}|$, we consider the problem of finding a minimal energy simultaneous eigenvector $\left|\Psi_{0}\right\rangle$ of both the Hamiltonian $\hat{H}$ and the number operator $\hat{N}$ such that $\hat{N}\left|\Psi_{0}\right\rangle=N\left|\Psi_{0}\right\rangle$ and $\hat{H}\left|\Psi_{0}\right\rangle=E_{0}\left|\Psi_{0}\right\rangle$. Such an eigenvector admits a characterization in terms of the Rayleigh quotient as follows,

$$
\left|\Psi_{0}\right\rangle \in \underset{\Psi \in \mathcal{H}_{N}: \Psi \neq 0}{\arg \min } \frac{\langle\Psi|\hat{H}| \Psi\rangle}{\langle\Psi \mid \Psi\rangle} .
$$

In the case of the interacting Hamiltonian (2), optimization over all $\mathrm{N}$-particle wave functions is intractable, so we focus on the simpler problem of selecting a wave function from a variational class of trial wave functions. A subset of $\mathcal{H}_{N}$ is chosen by specifying a family $\mathcal{F} \subseteq \mathcal{A}_{N}$ of antisymmetric functions. For each $f \in \mathcal{F}$, the associated wave function $\left|\Psi_{f}\right\rangle \in \mathcal{H}_{N}$ gives an upper bound on the ground-state energy,

$$
E_{0} \leqslant \frac{\left\langle\Psi_{f}|\hat{H}| \Psi_{f}\right\rangle}{\left\langle\Psi_{f} \mid \Psi_{f}\right\rangle}
$$

and thus optimizing this bound over $\mathcal{F}$ yields an approximation of the ground-state eigenpair.

By standard arguments reviewed in the Appendixes, the above Rayleigh quotient can be expressed as a classical expectation value of a local energy functional

$$
\frac{\left\langle\Psi_{f}|\hat{H}| \Psi_{f}\right\rangle}{\left\langle\Psi_{f} \mid \Psi_{f}\right\rangle}=\underset{x \sim \pi_{f}}{\mathbb{E}}\left[E_{f}(x)\right],
$$

\footnotetext{
${ }^{1}$ The domain of definition of $C$ follows from the fact that $L$ is assumed to be even.
} 
Algorithm 1 Markov Chain Metropolis

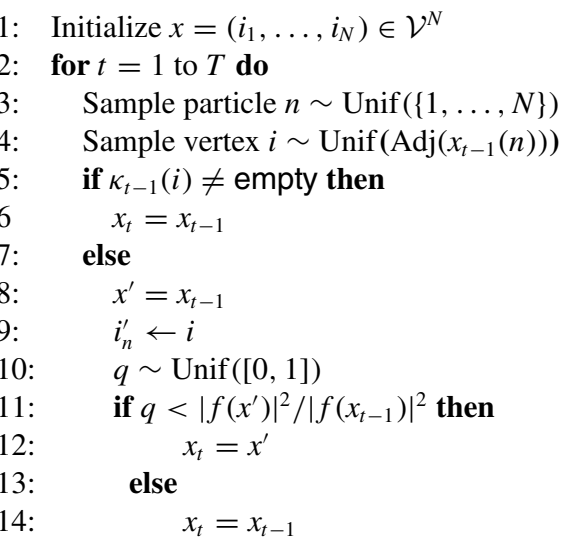

where $\pi_{f}$ is a permutation-invariant probability distribution over the classical state space $\mathcal{V}^{N}$, which assigns a probability to $x \in \mathcal{V}^{N}$ given by

$$
\pi_{f}(x)=\frac{|f(x)|^{2}}{\sum_{x^{\prime} \in \mathcal{V}^{N}}\left|f\left(x^{\prime}\right)\right|^{2}},
$$

and the local energy $E_{f}$ is a permutation-invariant function of $x \in \mathcal{V}^{N}$ defined by

$$
E_{f}(x)=-t \sum_{x^{\prime} \in \Delta_{x}} \frac{f\left(x^{\prime}\right)}{f(x)}+V \sum_{\{i, j\} \in \mathcal{E}} n_{i} n_{j},
$$

where $\Delta_{x} \subseteq \mathcal{V}^{N}$ denotes the set of classical states obtained by applying the kinetic operator to $|x\rangle$, and $n_{i} \in\{0,1\}$ denotes the binary occupation number of vertex $i \in \mathcal{V}$. In practice, the family of antisymmetric functions $\mathcal{F}$ is parametrized by unconstrained variational parameters $\theta \in \mathbb{R}^{d}$, and we locally optimize the following loss function:

$$
L(\theta):=\underset{x \sim \pi_{f_{\theta}}}{\mathbb{E}}\left[E_{f_{\theta}}(x)\right] .
$$

Sampling from the probability density $\pi_{f}$ was performed using a Markov chain Monte Carlo strategy outlined in Algorithm 1. The configuration space of the Markov chain is given by the classical state space $\mathcal{V}^{N}$. It is convenient to maintain a lookup table $\kappa: \mathcal{V} \rightarrow[N] \cup\{$ empty $\}$ which returns the location of a given vertex in the array $x=\left(i_{1}, \ldots, i_{N}\right)$, or empty if absent.

\section{B. Hartree-Fock}

It is instructive to contrast the VMC optimization problem with Hartree-Fock, which provides one of our baselines. Suppose that the matrix $\phi \in \mathbb{C}^{|\mathcal{V}| \times N}$ is an isometric matrix; that is, $\phi^{\dagger} \phi=\mathbb{1}_{N}$ and therefore $P=\phi \phi^{\dagger}$ is a Hermitian projection onto the image of $\phi$. If we define a family of $N$ creation operators,

$$
\widetilde{c}_{n}^{\dagger}=\sum_{i \in \mathcal{V}} \hat{c}_{i}^{\dagger} \phi_{n}(i),
$$

and define the $N$-particle normalized Hartree-Fock state,

$$
\left|\Psi_{\mathrm{HF}}\right\rangle=\widetilde{c}_{1}^{\dagger} \cdots \widetilde{c}_{N}^{\dagger}|0\rangle,
$$

then it is easily shown that the entries of the projection matrix $P \in \mathbb{C}^{|\mathcal{V}| \times|\mathcal{V}|}$ are given by

$$
P_{i j}=\left\langle\Psi_{\mathrm{HF}}\left|\hat{c}_{j}^{\dagger} \hat{c}_{i}\right| \Psi_{\mathrm{HF}}\right\rangle
$$

Expressing the Hamiltonian in terms of the adjacency matrix $A$ for the graph,

$$
\hat{H}=\sum_{(i, j) \in \mathcal{V}^{2}} A_{i j}\left[-t \hat{c}_{i}^{\dagger} \hat{c}_{j}-\frac{1}{2} V \hat{c}_{i}^{\dagger} \hat{c}_{j}^{\dagger} \hat{c}_{i} \hat{c}_{j}\right]
$$

where we have used the fact that $A$ is zero on the diagonal. The quantum expectation value of the Hamiltonian in the HartreeFock state is thus given by

$$
\left\langle\Psi_{\mathrm{HF}}|\hat{H}| \Psi_{\mathrm{HF}}\right\rangle=\sum_{(i, j) \in \mathcal{V}^{2}} A_{i j}\left[-t P_{j i}-\frac{1}{2} V\left(P_{i j} P_{j i}-P_{i i} P_{j j}\right)\right] .
$$

The optimization problem is to minimize the energy $E_{\mathrm{HF}}(\phi):=\left\langle\Psi_{\mathrm{HF}}|\hat{H}| \Psi_{\mathrm{HF}}\right\rangle$ viewed as a function of the isometric matrix $\phi$,

$$
\begin{aligned}
& \operatorname{minimize} E_{\mathrm{HF}}(\phi) \\
& \text { subject to } \phi^{\dagger} \phi=\mathbb{1}_{N} .
\end{aligned}
$$

In practice, we approximated the solution of the above constrained optimization problem by alternating between steps of the Adam optimization followed by orthogonalization of the columns of $\phi$. This kind of alternating optimization is described in Ref. [33].

\section{Wave-function ansatz}

Since the focus of this paper is ground-state optimization, we restrict ourselves to real-valued wave functions by exploiting the time-reversal invariance of the Hamiltonian. To describe our choice of antisymmetric functions $\mathcal{F}$, it is useful to define an indicator vector $n_{x} \in\{ \pm 1\}^{|\mathcal{V}|}$ for the configuration $x \in \mathcal{V}^{N}$, which is given in terms of the recentered binary occupations

$$
n_{x}:=\left(2 n_{i}-1\right)_{i \in \mathcal{V}},
$$

where $n_{i} \in\{0,1\}$. The family of antisymmetric functions $\mathcal{F}$ is chosen to consist of parametrized functions $f_{\theta}$ possessing a generalized Jastrow-Slater form, meaning that their dependence on the occupation numbers factorizes from a Slater determinant as follows:

$$
f_{\theta}(x)=\psi_{0}(x) J\left(n_{x}\right) S\left(n_{x}\right) .
$$

The domain and range of the constituent functions appearing in the above factorization is given as follows:

$$
\begin{gathered}
\psi_{0}: \mathcal{V}^{N} \rightarrow \mathbb{R}, \\
J:\{ \pm 1\}^{|\mathcal{V}|} \rightarrow[0, \infty), \\
S:\{ \pm 1\}^{|\mathcal{V}|} \rightarrow[-1,1] .
\end{gathered}
$$

In particular, $\psi_{0}$ is a Slater determinant, while $J$ and $S$ are neural networks, chosen with the property that they are invariant under a subset of lattice symmetries, and furthermore that they approach constant functions for some setting of the parameters. The proposed ansatz can thus be viewed as a 


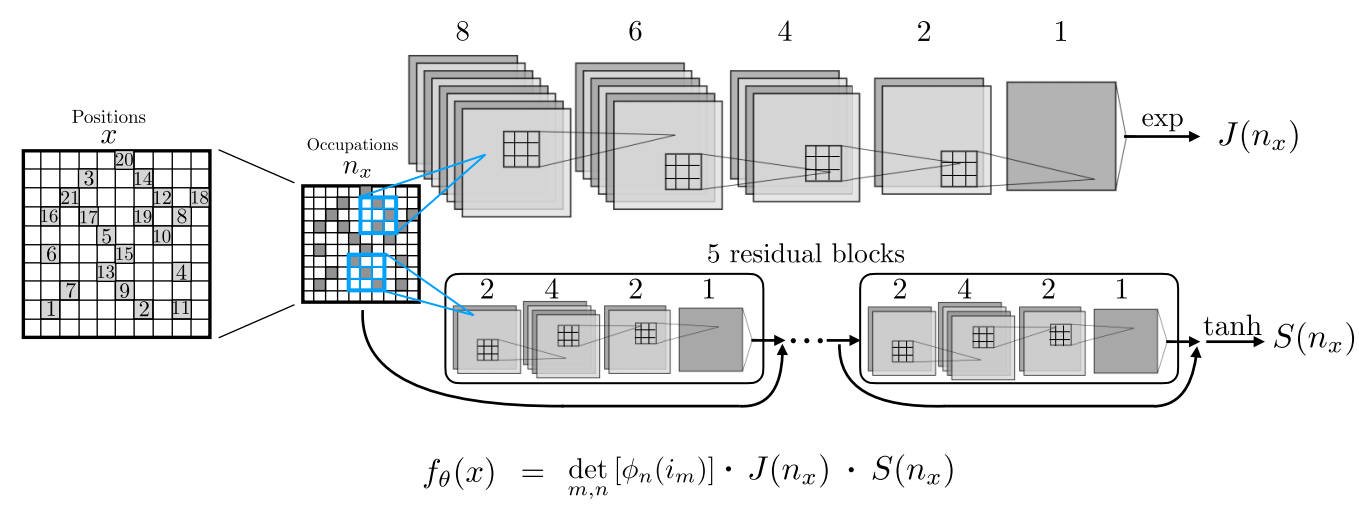

FIG. 1. Diagram of the wave-function ansatz as defined in Eq. (20). From left to right: square lattice of side length $L=10$ with 20 particles, occupation map in the lattice that the sign and amplitude networks take as an input. Top and bottom convolutional networks are amplitude and sign factors, respectively. The number of convolutional filters is indicated above each layer.

deformation of the Hartree-Fock wave function by a Jastrow factor and an additional factor that corrects the sign structure. The fact that $J$ and $S$ can exactly represent constant functions implies that the proposed ansatz is exact in the noninteracting $(V / t=0)$ and classical $(V / t \rightarrow \infty)$ limits. An ablative study conducted for small systems indeed found that dropping the $S$ network systematically decreased performance, indicating that the combination of the Slater determinant and strictly positive Jastrow factor is unable to capture the correct sign structure of the ground state. These results are deferred to the Supplemental Material [34].

The variational parameters characterizing the Slater determinant consist of a $|\mathcal{V}| \times N$ matrix $\phi=\left[\phi_{1}, \ldots, \phi_{N}\right] \in$ $\mathbb{R}^{|\mathcal{V}| \times N}$. Denoting the $i$ th entry of the $n$th column $\phi_{n} \in \mathbb{R}^{|\mathcal{V}|}$ by $\phi_{n}(i)$, it follows that the Slater determinant is $\psi_{0}(x)=$ $\operatorname{det}_{m, n}\left[\phi_{n}\left(i_{m}\right)\right]$ where the orbital functions $\phi_{n}: \mathcal{V} \rightarrow \mathbb{R}$ are neither normalized nor orthogonal.

The remaining variational parameters $\theta_{J} \in \mathbb{R}^{d_{J}}$ and $\theta_{S} \in$ $\mathbb{R}^{d_{S}}$ characterize the weights and biases of the neural networks $J$ and $S$. To meet the desiderata of translational invariance and ability to represent the constant function, we choose $J$ to be a convolutional feed-forward network with output exponential nonlinearity and we chose $S$ to be a deep residual network [35] with convolutional residual blocks, followed by an averaging layer, a fixed affine transformation, ${ }^{2}$ and final output tanh nonlinearity. In both cases the convolutions employed periodic boundary conditions (see Fig. 1).

The variational parameters $\theta=\left(\phi, \theta_{J}, \theta_{S}\right) \in \mathbb{R}^{d}(d=$ $|\mathcal{V}| N+d_{J}+d_{S}$ ) were jointly optimized by using the stochastic reconfiguration method [36], which can be interpreted as imaginary time evolution or a particular case of the natural gradient optimization $[37,38]$.

The variational parameters $\left(\theta_{J}, \theta_{S}\right)$ were initialized by using standard random initialization strategies and the Slater determinant parameters $\phi$ were initialized by using the solution of the Hartree-Fock optimization scheme described above to improve convergence.

The number of variational parameters in the neural networks do not change significantly with system size as a result

\footnotetext{
${ }^{2}$ In practice, we chose the affine layer to be a pointwise application of $x \mapsto \frac{x}{2(2 N-|\mathcal{V}|)}+\frac{1}{2}$.
}

of using convolutions with a fixed number of channels and filter size. It follows that the largest contribution to the number of parameters (filters) does not change with system size. Although the number of biases scales linearly with system size, for the system sizes considered, the number of biases is a small fraction of the total number of parameters. This scaling is comparable to typical Jastrow factors invariant under translations, where the number of parameters also scales linearly with the system size.

\section{RESULTS}

In this section we analyze the ground-state correlation functions and the phase structure by performing ground-state optimization of the neural network ansatz using variational Monte Carlo. In particular, two-point density correlation functions are analyzed to probe the nature of the charge-ordered phase. Then the phase diagram is constructed by performing a finite-size scaling analysis of the density order parameter (3).

\section{A. Exact diagonalization benchmark}

To verify the implementation, the exact ground-state eigenpair was determined by using the Lanczos algorithm as implemented in QUSPIN $[39,40]$, working in the zero-momentum sector containing the ground state. Exact diagonalization is practical for system sizes up to $L=6$ with $N \leqslant 17$ particles.

The relative error in the ground-state energy obtained using VMC optimization of the neural network is shown in Fig. 2, alongside the relative error obtained using unrestricted Hartree-Fock for comparison. Unsurprisingly, the neural-network ansatz outperforms the unrestricted HF in terms of accuracy of ground-state energy, with relative errors smaller by up to two orders of magnitude for certain values of the coupling. For most values of $V / t$, the relative error does not exceed $O\left(10^{-3}\right)$. In the worst cases, which correspond to large values of the interaction (but still far from the classical limit), the relative error is no larger than $O\left(10^{-2}\right)$. The ground-state energies obtained using our method were found to be lower than those obtained using tensor-product projected states [29] and string bond states [28], which are available for system size $L=4$ and half occupation $(\bar{n}=0.5)$. 

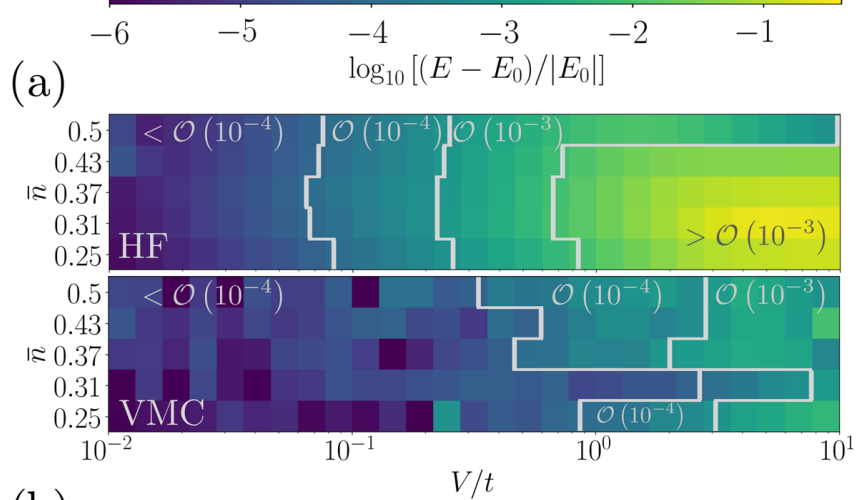

(b)



(c)
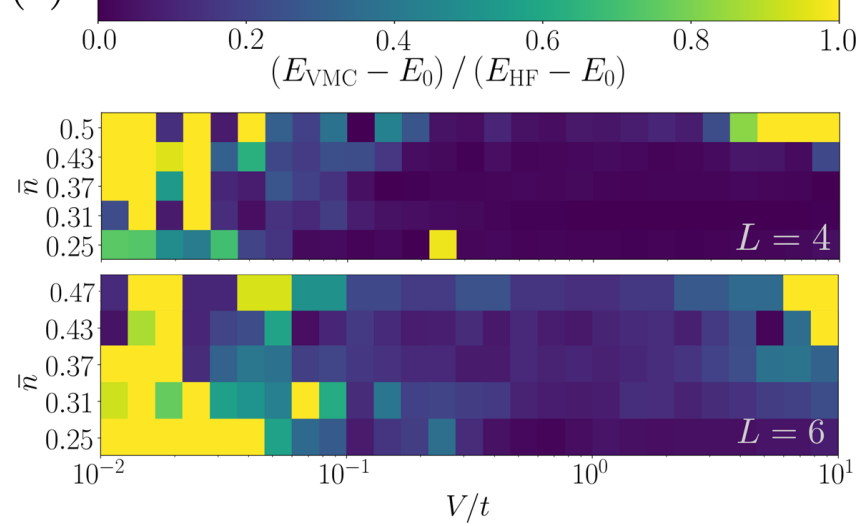

FIG. 2. Benchmark of the proposed variational ansatz (VMC) and Hartree-Fock approximation (HF) using the exact ED states. (a), (b) Color-maps show the relative error of the energy in the square lattice of size (a) $L=4$ and in the square lattice of size (b) $L=6$. Relative error is shown at different fillings of the lattice and values of the coupling constant. Lines separate regions of the phase diagram with different orders of magnitude of the relative error as indicated. (c) Shows the error of the proposed ansatz in panels (a) and (b), relative to the error of the Hartree-Fock approximation, in the square lattice of size $L=4$ and $L=6$.

To benchmark the ground-state wave function beyond the energy error, we computed density-density correlations as defined in Eq. (5) and compared against ED results, as shown in Fig. 3. The neural-network ansatz accurately reproduces the exact-correlation functions for any graph distance $r$ at any $V / t$ and filling values.

The benchmark with ED shows that the proposed neuralnetwork variational ansatz provides an accurate approxima-

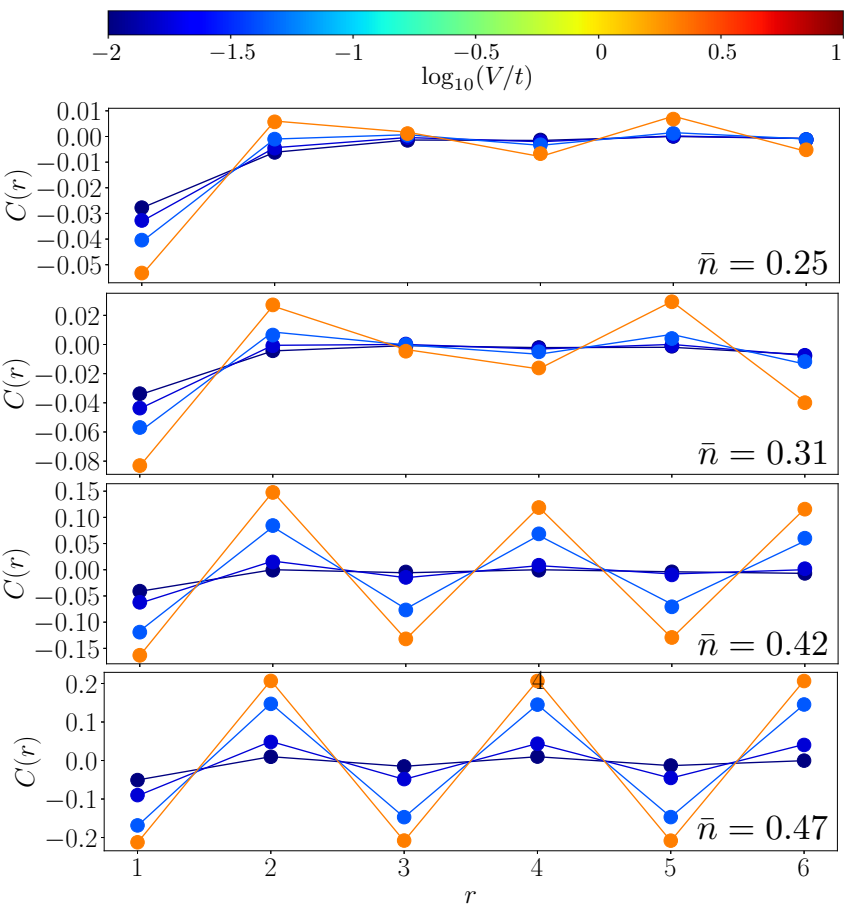

FIG. 3. Benchmark of the proposed variational ansatz (VMC) using ED eigenpairs in the square lattice of side length $L=6$. Two-point density correlation functions [Eq. (5)] are shown as a function of graph distance $r$ at different values of $V / t: V / t=0.01$, $V / t=0.599, V / t=2.15$, and $V / t=5.99$, as shown by the color scale in the top of the panel. Solid lines are the correlations from ED and the dots correspond to the correlations computed with our approach. Different panels correspond to different fillings, as indicated in each panel. The results obtained using ED and neural networks are visually indistinguishable.

tion to the ground-state, making it a suitable tool to study the phase diagram of the model.

\section{B. Phase diagram}

\section{Correlation functions}

The ground-state two-point density correlation functions are shown for the largest available system size $(L=10)$ in Fig. 4(a). In practice, computation times did not change significantly between the $6 \times 6$ and $10 \times 10$ systems, and we expect, as a result of translational invariance, that the results should generalize to larger system sizes, given additional training time.

For weak coupling, the correlation functions barely oscillate and decay to zero with increasing graph distance $r$ between correlation pairs. As the interaction strength increases, the correlations spatially oscillate with increasing amplitude due to ordering of the charge distribution when the transition is crossed. In the charge-ordered phase, the amplitude of the oscillations decays (as a function of $r$ ) at a slower rate than in the metallic phase. At half occupation the charge ordering is staggered, leading to commensurate oscillations in the correlation functions. Away from half occupation, due to the geometry of the lattice, the charge order cannot be staggered, leading to incommensurate oscillations 




FIG. 4. Two-point density correlation function defined in Eq. (5) and the moduli of their renormalized Fourier modes defined in Eq. (6). Different colors indicate different values of $V / t$, as indicated in the legend in the top of the plot. (a) Two-point density correlation functions as a function of the graph distance $r$. System size is $L=10$. Dots represent the obtained values for the correlations and black solid lines are for visual guidance. Each panel corresponds to a different filling as indicated. (b) Modulus of the Fourier transform of the correlation functions displayed in the corresponding (a) panels, as a function of $k$. Note that $\left|\widetilde{C}_{\text {ren }}(k)\right|$ is only shown for $k \in[0, \pi]$ as $\left|\widetilde{C}_{\text {ren }}(k)\right|$ is symmetrical with respect to $k=\pi$. (c) Amplitude peak in $\left|\widetilde{C}_{\text {ren }}(k)\right|$ shown in panel (a) as a function of the lattice size. For different system sizes the peak is identified as the maximum of $\left|\widetilde{C}_{\text {ren }}(k)\right|$ at the largest value of $V / t$ considered. Different panels correspond to different lattice fillings, as indicated.

in the correlations. For all occupations, increasing the interaction strength increases the amplitude of the oscillations, without significantly altering their wave form.

This behavior is also evident in the Fourier component amplitudes $\left|\widetilde{C}_{\text {ren }}(k)\right|$ [Fig. 4(b)]. For all values of occupation and for weak interaction, $\left|\widetilde{C}_{\text {ren }}(k)\right|$ exhibits a uniform distribution without peaks. When the coupling is large enough, the system transitions to the charge-ordered state where a peak appears in which the amplitude monotonically increases with $V / t$. Note that at $\bar{n}=0.24$ the peak is not well resolved due to the small number of $k$ values accessible in this system size. The position of the peak depends on the filling, as anticipated. At half occupation the peak is narrow and centered around $k=\pi$ (staggered ordering). Close to half occupation and for the considered system size, the peak is still centered at $k=\pi$ but with increased width due to the rise of noncommensurate order. Lower values of the filling lead to Fourier peaks that correspond to longer-wavelength orderings.

The results discussed above appear to be consistent with the formation of a CDW state as opposed to a phase-separated one. We further analyze the nature of the $\left|\widetilde{C}_{\text {ren }}(k)\right|$ peak by studying its amplitude as a function of the system size, as shown in Fig. 4(c). At values of $V / t$ corresponding to a metallic state, the amplitude of the corresponding Fourier mode saturates to a constant value, or decreases as $L$ is increased, depending on the filling. In contrast, in the CDW phase the amplitude of the peak increases with $L$. Although this scaling could in principle be used to determine the transition points, the system sizes we considered are not sufficient to accurately extract the critical point. This is in part due to the access to a limited set of Fourier modes in the discrete Fourier transform, which may not provide the necessary resolution in $k$ space to resolve the true period of the oscillations away from half filling. Despite these difficulties, the above results are consistent with a CDW phase [27], rather than a phase-separated one.

\section{Order parameter}

Figure 5 shows the order parameter (3) as a function of $V / t$ at different lattice occupations. Each panel also displays the order parameter at different system sizes $L=6, L=8$, and $L=10$. Interpolation is required to obtain the value of $o[\rho]$ at the desired filling at a given system size. Linear interpolation is used instead of higher-order interpolation schemes. The reason for this is that the order parameter takes values close to zero in the metallic phase, leading to negative values of the interpolated value of $o[\rho]$ when using higher-order methods. The order parameter takes a small but nonzero value in the metallic phase (smaller values of $V / t$ ) and abruptly increases upon crossing to the charge-ordered phase. In the metallic phase and at fixed values of the filling and coupling, $o[\rho] \geqslant 0$ decreases with system size. This is consistent with the CDW coming from the opening of a gap like in the $d=1$ case, where the amplitude of the charge-density wave increases with the magnitude of the gap [31]. In finite-sized systems the metallic phase has a small but nonzero gap, which decreases with the increase of system size and becomes zero in the thermodynamic limit. This nonzero gap leads to a small amplitude in the charge order.

Finite-size scaling is thus required to find the transition points. At fixed values of the density we analyze the order parameter as a function of $V / t$, starting in the metallic phase, where its value decreases with $L$. The transition point is taken where the order-parameter curves corresponding to different system sizes cross each other upon increasing the value of the coupling. Transition points are determined by the average of the first three crossing points. Error bars are determined by 

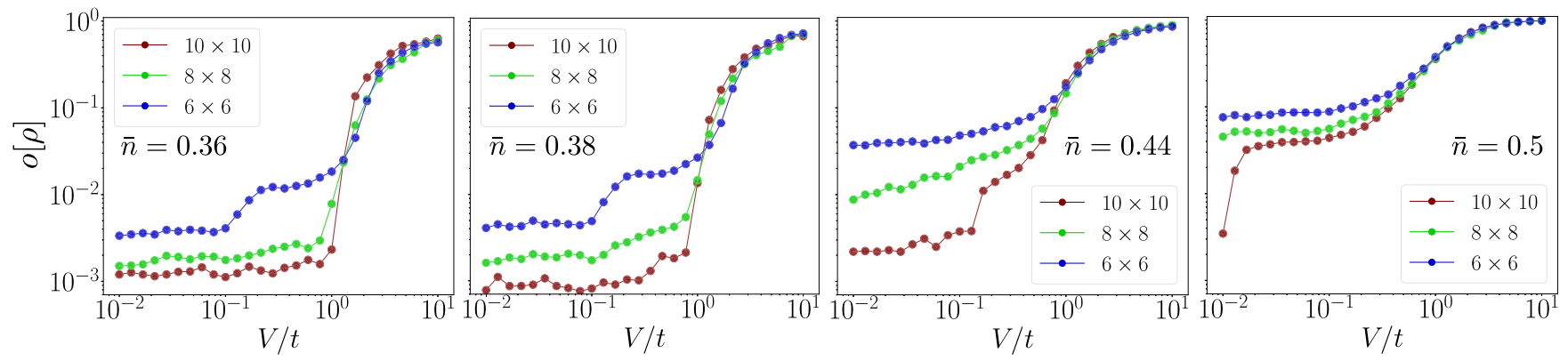

FIG. 5. Order parameter as defined in Eq. (3) as a function of the coupling constant. Each panel shows the order parameter in system sizes $L=6, L=8$, and $L=10$, as indicated in the legend with different colors. Lines connecting dots are for visual guidance. Different panels correspond to different fillings as indicated.

the maximum between the range of $V / t$ between the first three crossing points and the separation of the $V / t$ values sampled. The scaling of the order parameter at half filling is consistent with a transition at an infinitesimally small value of $V / t$ due to Fermi-surface nesting [41].

The phase diagram arising from these transition points is displayed in Fig. 6. Particle-hole symmetry has been applied to determine the phase boundary for $\bar{n}>0.5$ from the results obtained at $\bar{n}<0.5$.

\section{Phase diagram}

We conclude this study by analyzing the obtained phase diagram and comparing it with the phase diagram obtained with unrestricted Hartree-Fock [26] and IPEPS [30], in the grand canonical ensemble. Figure 6 shows the phase diagrams obtained with unrestricted HF [26], IPEPS [30], and with our neural-network ansatz superimposed onto a color map of the order parameter in the largest system size considered. In the IPEPS study [30], it was found that, by increasing the bond dimension (and consequently the accuracy of the ground-state approximation), the transition line shifts to higher values of $V / t$. The phase boundary obtained in this work lies at slightly larger values of the interaction than those obtained from IPEPS, which may indicate that the proposed neural-network ansatz is more accurate than the IPEPS wave function.

The magnitude of the order parameter in the largest system size analyzed $L=10$ already provides a good indication of the transition point as shown by the superimposed color map.

\section{CONCLUSION}

We showed that neural networks can be used to analyze the ground-state properties of lattice fermionic systems in first quantization. The proposed wave function and minimization scheme are applicable to arbitrary lattice models. In particular, we applied it to the study of the phase diagram of the twodimensional periodically identified square lattice with nearest neighbor repulsive interactions.

The exact diagonalization benchmarks demonstrate that the proposed wave function accurately captures the ground-state

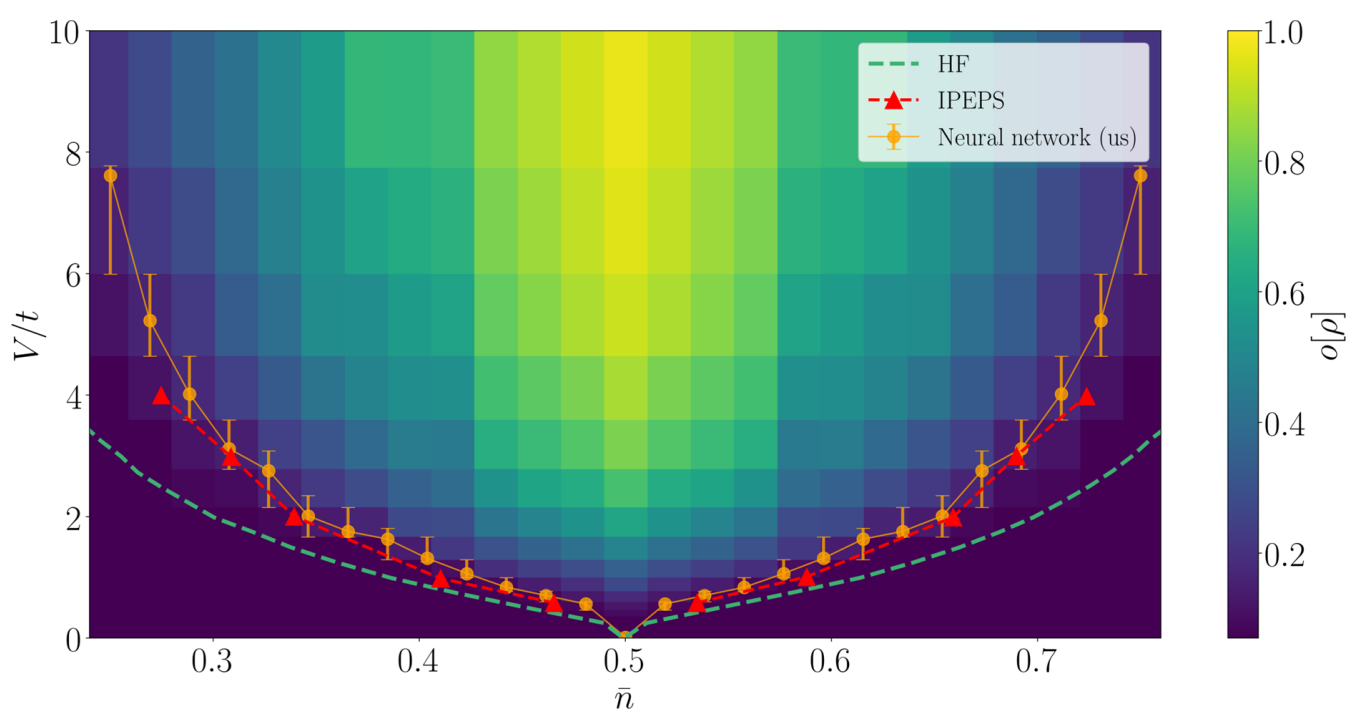

FIG. 6. Phase diagram of the two-dimensional interacting spinless fermion model under consideration. The Hartree-Fock and IPEPS transition lines are from Ref. [30]. The orange dots correspond to the transition points from the finite-size scaling of $o[\rho]$, using the proposed variational ansatz. Lines connecting the dots are for visual guidance. The color-map represents the CDW order parameter $o[\rho]$ in the largest system size studied $(L=10)$ at different values of the particle density and interaction strength. 
energy for a wide range of lattice fillings and interaction strengths. It also achieves lower energies than other approaches at half occupation for system size $L=4$ where comparison data are available. Furthermore, we tested the accuracy in reproducing other observables such as two-point density correlation functions finding essentially the same values as those from exact diagonalization.

The study of the two-point density correlation functions shows results consistent with a charge-density-wave state for large values of the coupling, rather than a phase separated one. A finite-size scaling analysis of the order parameter allowed us to obtain the phase boundaries for the model, allowing the construction of the phase diagram from a canonical ensemble approach.

\section{ACKNOWLEDGMENTS}

J.R.M. acknowledges support from the CCQ graduate fellowship in computational quantum physics. The Flatiron Institute is a division of the Simons Foundation. The authors acknowledge stimulating discussions with Antoine Georges, Risi Kondor, Erik Thiede, and Lei Wang.
[1] S. R. White, Density Matrix Formulation for Quantum Renormalization Groups, Phys. Rev. Lett. 69, 2863 (1992).

[2] S. R. White, Density-matrix algorithms for quantum renormalization groups, Phys. Rev. B 48, 10345 (1993).

[3] M. Fannes, B. Nachtergaele, and R. F. Werner, Finitely correlated states on quantum spin chains, Commun. Math. Phys. 144, 443 (1992).

[4] D. Pérez-García, F. Verstraete, M. M. Wolf, and J. I. Cirac, Matrix product state representations, Quantum Inf. Comput. 7, 401 (2007).

[5] M. B. Hastings, An area law for one-dimensional quantum systems, J. Stat. Mech. (2007) P08024.

[6] G. Carleo, I. Cirac, K. Cranmer, L. Daudet, M. Schuld, N. Tishby, L. Vogt-Maranto, and L. Zdeborová, Machine learning and the physical sciences, Rev. Mod. Phys. 91, 045002 (2019).

[7] G. Carleo and M. Troyer, Solving the quantum many-body problem with artificial neural networks, Science 355, 602 (2017).

[8] W. L. McMillan, Ground state of liquid $\mathrm{He}^{4}$, Phys. Rev. 138, A442 (1965).

[9] K. Choo, T. Neupert, and G. Carleo, Two-dimensional frustrated $J_{1}-J_{2}$ model studied with neural network quantum states, Phys. Rev. B 100, 125124 (2019).

[10] F. Ferrari, F. Becca, and J. Carrasquilla, Neural Gutzwillerprojected variational wave functions, Phys. Rev. B 100, 125131 (2019).

[11] Y. Nomura and M. Imada, Dirac-type nodal spin liquid revealed by machine learning, arXiv:2005.14142.

[12] T. Vieijra, C. Casert, J. Nys, W. De Neve, J. Haegeman, J. Ryckebusch, and F. Verstraete, Restricted Boltzmann Machines for Quantum States with Non-Abelian or Anyonic Symmetries, Phys. Rev. Lett. 124, 097201 (2020).

[13] K. Choo, A. Mezzacapo, and G. Carleo, Fermionic neuralnetwork states for ab-initio electronic structure, Nat. Commun. 11, 2368 (2020).

[14] Y. Nomura, A. S. Darmawan, Y. Yamaji, and M. Imada, Restricted Boltzmann machine learning for solving strongly correlated quantum systems, Phys. Rev. B 96, 205152 (2017).

[15] T. Misawa, S. Morita, K. Yoshimi, M. Kawamura, Y. Motoyama, K. Ido, T. Ohgoe, M. Imada, and T. Kato, mVMCopen-source software for many-variable variational Monte Carlo method, Comput. Phys. Commun. 235, 447 (2019).
[16] D. Luo and B. K. Clark, Backflow Transformations Via Neural Networks for Quantum Many-Body Wave Functions, Phys. Rev. Lett. 122, 226401 (2019).

[17] D. Pfau, J. S. Spencer, A. G. de G. Matthews, and W. M. C. Foulkes, Ab-initio solution of the many-electron Schrödinger equation with deep neural networks, Phys. Rev. Research 2, 033429 (2020).

[18] J. Hermann, Z. Schätzle, and F. Noé, Deep neural network solution of the electronic Schrödinger equation, Nat. Chem. 12, 891 (2020).

[19] D. Ceperley, G. V. Chester, and M. H. Kalos, Monte Carlo simulation of a many-fermion study, Phys. Rev. B 16, 3081 (1977).

[20] T. Westerhout, N. Astrakhantsev, K. S. Tikhonov, M. I. Katsnelson, and A. A. Bagrov, Generalization properties of neural network approximations to frustrated magnet ground states, Nat. Commun. 11, 1593 (2020).

[21] J. E. Gubernatis, D. J. Scalapino, R. L. Sugar, and W. D. Toussaint, Two-dimensional spin-polarized fermion lattice gases, Phys. Rev. B 32, 103 (1985).

[22] D. J. Scalapino, R. L. Sugar, and W. D. Toussaint, Monte Carlo study of a two-dimensional spin-polarized fermion lattice gas, Phys. Rev. B 29, 5253 (1984).

[23] R. H. McKenzie, J. Merino, J. B. Marston, and O. P. Sushkov, Charge ordering and antiferromagnetic exchange in layered molecular crystals of the $\theta$ type, Phys. Rev. B 64, 085109 (2001).

[24] A. Moreo and D. J. Scalapino, Cold Attractive Spin Polarized Fermi Lattice Gases and the Doped Positive $U$ Hubbard Model, Phys. Rev. Lett. 98, 216402 (2007).

[25] G. B. Partridge, W. Li, R. I. Kamar, Y.-A. Liao, and R. G. Hulet, Pairing and phase separation in a polarized Fermi gas, Science 311, 503 (2006).

[26] W. R. Czart, S. Robaszkiewicz, and B. Tobijaszewska, Charge ordering and phase separations in the spinless fermion model with repulsive intersite interaction, Acta Phys. Pol. 114, 129 (2008).

[27] J. de Woul and E. Langmann, Partially gapped fermions in 2D, J. Stat. Phys. 139, 1033 (2010).

[28] J.-P. Song and R. T. Clay, Monte Carlo simulations of two-dimensional fermion systems with string-bond states, Phys. Rev. B 89, 075101 (2014). 
[29] O. Sikora, H.-W. Chang, C.-P. Chou, F. Pollmann, and Y.-J. Kao, Variational Monte Carlo simulations using tensor-product projected states, Phys. Rev. B 91, 165113 (2015).

[30] P. Corboz, R. Orús, B. Bauer, and G. Vidal, Simulation of strongly correlated fermions in two spatial dimensions with fermionic projected entangled-pair states, Phys. Rev. B 81, 165104 (2010).

[31] T. Giamarchi, in Quantum Physics in One Dimension (Oxford University Press, Oxford, 2003), pp. 160-199.

[32] L. Wang, P. Corboz, and M. Troyer, Fermionic quantum critical point of spinless fermions on a honeycomb lattice, New J. Phys. 16, 103008 (2014).

[33] F. Becca and S. Sorella, Quantum Monte Carlo Approaches for Correlated Systems (Cambridge University Press, Cambridge, England, 2017).

[34] See Supplemental Material at http://link.aps.org/supplemental/ 10.1103/PhysRevB.102.205122 for comparison between signed and unsigned networks.
[35] K. He, X. Zhang, S. Ren, and J. Sun, Deep residual learning for image recognition, arXiv:1512.03385.

[36] S. Sorella, M. Casula, and D. Rocca, Weak binding between two aromatic rings: Feeling the van der Waals attraction by quantum Monte Carlo methods, J. Chem. Phys. 127, 014105 (2007).

[37] S.-I. Amari, Natural gradient works efficiently in learning, Neural Comput. 10, 251 (1998).

[38] J. Stokes, J. Izaac, N. Killoran, and G. Carleo, Quantum natural gradient, Quantum 4, 269 (2020).

[39] P. Weinberg and M. Bukov, QuSpin: A Python package for dynamics and exact diagonalisation of quantum many body systems Part I: Spin chains, SciPost Phys. 2, 003 (2017).

[40] P. Weinberg and M. Bukov, QuSpin: A Python package for dynamics and exact diagonalisation of quantum many body systems. Part II: Bosons, fermions and higher spins, SciPost Phys. 7, 020 (2019).

[41] M. D. Johannes and I. I. Mazin, Fermi surface nesting and the origin of charge density waves in metals, Phys. Rev. B 77, 165135 (2008). 